\title{
Effects of fisetin on mouse lipid metabolism in vitro and in vivo
}

\author{
Tomoaki Yonesaka ${ }^{1}$, Kaoru Yoshida ${ }^{2}$, Shunsuke Iizuka ${ }^{1}$, Hiromi Hagiwara ${ }^{1}$ \\ ${ }^{1}$ Department of Biomedical Engineering, ${ }^{2}$ Biomedical Engineering Center, Toin University of \\ Yokohama, 1614 Kurogane-cho, Aoba-ku, Yokohama 225-8502, Japan
}

Corresponding author: Hiromi Hagiwara, $\mathrm{PhD}$, Department of Biomedical Engineering, Toin University of Yokohama, 1614 Kurogane-cho, Aoba-ku, Yokohama 225-8502, Japan

Submission date: August 27, 2014; Acceptance date: October 15, 2014; Publication date:

October 17, 2014

\begin{abstract}
:
Objective: The aim of this study was to investigate the anti-obesity effects of the polyphenol fisetin in 3T3-L1 preadipocytes and C57BL/6 female mice that were fed a high-fat diet (HFD).

Background: Polyphenols, such as sakuranetin, hesperetin, tea catechin, and quercetin, reportedly regulate adipocyte differentiation in 3T3-L1 cells. Furthermore, green tea, apple, and molokheiya polyphenols exhibit anti-obesity activities in HFD-treated obese rats or mice. Fisetin is abundant in plants, fruits, and vegetables and exhibits multiple biological activities, such as the inhibition of prostate cancer growth, neuroprotection, and protection against osteoporosis. In addition, fisetin regulates obesity by targeting mammalian target of rapamycin complex 1 signaling, which is a central mediator of lipid biosynthesis.
\end{abstract}

Materials and methods: (1) in vitro experiments; we investigated the effects of fisetin on intracellular lipid accumulation and glycerol-3-phosphate activity during the differentiation of 3T3-L1 cells. We monitored expression of adipogenetic related-genes in 3T3-L1 cells by real-time polymerase-chain-reaction. (2) in vivo experiments; we examined the effects of fisetin on anti-obesity activities in C57BL/6 female mice that were fed a HFD. 
Results: Fisetin inhibited intracellular lipid accumulation and glycerol-3-phosphate activity during the differentiation of 3T3-L1 cells in a dose-dependent manner (50-75 $\mu \mathrm{M})$. In addition, real-time polymerase-chain-reaction revealed that this compound suppressed the expression of peroxisome proliferator-activated receptor $\gamma$ (PPAR $\gamma$ ), adipocyte protein 2, and perilipin mRNAs in 3T3-L1 cells. In contrast, anti-obesity activities, such as reduction of body weight and fat tissue, and improvements in obesity-related blood biochemical parameters and fatty liver, were not observed in HFD-induced mice treated with fisetin $(20 \mathrm{mg} / \mathrm{kg}$ body weight $)$ by intraperitoneal injections twice per week for 8 weeks.

Conclusions: Fisetin exerted anti-adipogenic activities by inhibiting the expression of PPAR $\gamma$ mRNA in 3T3-L1 preadipocytes. However, fisetin $(20 \mathrm{mg} / \mathrm{kg}$ body weight $)$ did not affect HFD-induced obesity. Our findings indicated that fisetin could be used as an effective remedy in the treatment of the symptoms of obesity.

Keywords: Fisetin, MC3T3-L1 cells, Fatty accumulation, PPAR $\gamma$, Obesity, Anti-obesity activity

\section{INTRODUCTION:}

Obesity and related metabolic diseases such as type- 2 diabetes, atherosclerosis, hypertension, and fatty liver, are the most prevalent nutrition-related issues in the world. The control of adipocyte differentiation and proliferation could be a strategy for the prevention and treatment of obesity and related metabolic diseases. The differentiation of preadipocyte into adipocyte is controlled by a complex network of a variety of proteins. For example, it has been shown that peroxisome proliferator-activated receptor $\gamma(\operatorname{PPAR} \gamma)$ plays critical roles in adipocyte differentiation [1, 2]. Increased oxidative stress in accumulated fat is an early cause of metabolic syndrome and the redox state in adipose tissue is a potentially useful therapeutic target for obesity related metabolic diseases [3]. Polyphenols display anti-oxidant and anti-inflammatory properties. Recent reports have stated that sakuranetin [4], hesperetin [5, 6], and naringenin [6] induce adipogenesis in 3T3-L1 cells. In contrast, quercetin [7] and epigallocatechin gallate [8] exhibit anti-obesity activities in 3T3-L1 preadipocytes. Kaempferitrin also inhibits glucose transporter 4 translocation and glucose uptake in 3T3-L1 adipocytes [9]. Furthermore, green tea [10], apple [11], and molokheiya [12] polyphenols show anti-obesity activities in high-fat diet (HFD)-treated obese rats or mice. 
Fisetin $(3,7,3$ ', 4 '-tetrahydroxyflavone) is a polyphenol that is abundant in plants, fruits, and vegetables and exhibits multiple biological activities, such as inhibition of prostate cancer growth [13], prevention of rheumatoid arthritis [14], and protection against osteoporosis [15]. Furthermore, fisetin promotes ERK-dependent long-term potentiation in rat hippocampal slices and enhances memory in mice [16]. Fisetin is also known to act via the activation of the Nrf2 pathway [17], which is the major regulator of cytoprotective responses to oxidative and electrophilic stress. Recently, it has been reported that fisetin regulates obesity by targeting mammalian target of rapamycin complex 1 signaling, which is a central mediator of lipid biosynthesis [18].

The present study was designed to evaluate the in vitro and in vivo anti-obesity effects of fisetin in 3T3-L1 adipocytes and HFD-treated mice. Our results indicated that fisetin attenuated adipogenesis in 3T3-L1 cells by inhibiting the expression of PPAR $\gamma$ mRNA.

\section{MATERIALS AND METHODS:}

Materials: Fisetin was purchased from Tokyo Kasei Co., Ltd. (Tokyo, Japan). Dulbecco's Modified Eagle's Medium (DMEM; High-glucose) and penicillin/streptomycin antibiotic mixture were obtained from Sigma-Aldrich K.K. (Tokyo, Japan) and Life Technologies Corporation (Grand Island, NY, USA), respectively. Fetal bovine serum was obtained from JRH Bioscience, Inc. (Lenexa, KS, USA).

Cell culture: Mouse preadipocyte 3T3-L1 cells were obtained from the RIKEN Cell Bank (Tsukuba, Japan). Cells were maintained in a $55-\mathrm{cm}^{2}$ dish in DMEM, which was supplemented with $10 \%$ fetal bovine serum, 50 units $/ \mathrm{mL}$ penicillin, and $50 \mu \mathrm{g} / \mathrm{mL}$ streptomycin, in a humidified atmosphere of $5 \% \mathrm{CO}_{2}$ in air at $37{ }^{\circ} \mathrm{C}$. After reaching $70 \%$ confluence, the cells were detached by treatment with $0.05 \%$ trypsin, replated in either 6-well plates (area of each well, $9.4 \mathrm{~cm}^{2}$ ) or 12-well plates (area of each well, $3.8 \mathrm{~cm}^{2}$ ) at a density of $2 \times 10^{4} \mathrm{cells} / \mathrm{cm}^{2}$, and grown in DMEM, which was supplemented with $10 \%$ fetal bovine serum, 50 units/mL penicillin, and $50 \mu \mathrm{g} / \mathrm{mL}$ streptomycin. After confluence, the cells were cultured for 2 days in the presence of various concentrations of fisetin, and continuously cultured for 2 days with $0.25 \mu \mathrm{M}$ of dexamethasone (Sigma-Aldrich K.K.), $0.2 \mathrm{mM}$ 3-iso-butyl-1-methylxanthine (Sigma-Aldrich K.K.), and $10 \mu \mathrm{g} / \mathrm{mL}$ insulin (Wako Pure Chemical Industries, Ltd., Osaka, Japan)(DMI induction). The cells were then cultured in medium containing $5 \mu \mathrm{g} / \mathrm{mL}$ of insulin. 
Animal models: Female 4-week-old C57BL/6J mice were obtained from CLEA Japan, Inc. (Tokyo, Japan). The mice were randomly divided into the following four groups: mice fed the Mouse Feed (MF; Oriental Yeast Co. Ltd., Tokyo, Japan) with the administration of saline (normal group), mice fed the MF with the administration of fisetin $[20 \mathrm{mg} / \mathrm{kg}$ body weight, twice per a week, intraperitoneal (i.p.)] (normal fisetin group), mice fed a HFD (CLEA Rodent Diet Quick Fat; CLEA Japan, Inc.) with the administration of saline (HFD group), and mice fed a HFD with the administration of fisetin $(20 \mathrm{mg} / \mathrm{kg}$ body weight, twice per a week, i.p.) (HFD-fisetin group) for 8 weeks. Body weight was measured weekly. The care and use of the animals followed our institutional and national guidelines, and all of the experimental procedures were approved by the animal committee of Toin University of Yokohama.

Computed Tomography (CT) Scan Analysis: The amount of visceral fat and subcutaneous fat in the HFD-fed mice were examined by radiography with a CT apparatus (LaTheta LCT-100M, Hitachi Aloka Medical, Ltd, Tokyo, Japan), according to the manufacturer's protocol. The CT scanning was done at 1-mm intervals from the diaphragm to the bottom of the abdominal cavity. First, an overview scan of the whole mouse was conducted to allow for the selection of the regions of interest for the different scans. To quantify the visceral and subcutaneous fat depots the area between the proximal end of lumbar vertebra L3 and the distal end of L5 was scanned. For the liver fat quantification the areas that include liver and spleen (between the cranial part of the diaphragm and the lumbar vertebra L3) and adipose tissue (narrow area in the region of the lumbosacral joint) were scanned. Abdominal muscle was used as a discriminant between the visceral and subcutaneous fat depots.

Sample preparation and procedures: Eight weeks after supplementation with the experimental diets, the mice were sacrificed. Blood samples were collected from the heart and centrifuged at $1,000 \times g$ for $10 \mathrm{~min}$, and serum triglyceride (TG), total cholesterol, asparatate aminotransferase (AST), and alanine aminotransferase (ALT) levels were measured by BML, Inc. (Tokyo, Japan). The liver and kidney were excised and fixed in a 10\% neutral-buffered formalin solution and processed for histology. The liver and kidney were snap-frozen with Optimal Cutting Temperature (OCT) compound in liquid nitrogen and stored at $-80^{\circ} \mathrm{C}$.

Histological analysis: Fixed tissues were embedded in paraffin, sliced into $4-\mu \mathrm{m}$-thick sections and stained with hematoxylin and eosin. The pathological changes were assessed and 
photographed with the All-in-One Fluorescence Microscope system (BZ-8100 and BZ-Analyzer, Keyence Corporation, Osaka, Japan).

Oil-red $\boldsymbol{O}$ staining: Cells were cultured in medium containing fisetin $(0,25,50$, or $75 \mu \mathrm{M})$ for 2 days. Frozen liver sections and cells were fixed with $4 \%$ paraformaldehyde for $1 \mathrm{~h}$, and then stained with $3 \mathrm{mg} / \mathrm{mL}$ Oil-red $\mathrm{O}$ (in $60 \%$ isopropanol) for $10 \mathrm{~min}$. After washing twice with $60 \%$ isopropanol, images were collected on the All-in-One Fluorescence Microscope system. In addition, the dye was eluted for $10 \mathrm{~min}$ with $100 \%$ isopropanol. The concentration of the eluted dye was determined from measurements of absorbance (Optical Density, $420 \mathrm{~nm}$ ).

Glycerol-3-phosphate dehydrogenase (GPDH) activity: GPDH activity was measured with a GPDH Activity Assay Kit (Takara Bio, Inc., Tokyo, Japan) according to the manufacturer's instructions. One unit was defined as the amount of enzyme that was required for the consumption of $1 \mu \mathrm{mol}$ of nicotinamide adenine dinucleotide in $1 \mathrm{~min}$ at $30^{\circ} \mathrm{C}$. Each assay was conducted in duplicate.

Real-time polymerase-chain-reaction (PCR): The mRNA levels of expression of PPAR $\gamma$, adipocyte protein $2(\mathrm{aP} 2)$, and perilipin in 3T3-L1 cells were examined by real-time PCR. The RNeasy Mini Kit (Qiagen K.K., Tokyo, Japan) was used to extract RNA from the cells that had been exposed to various concentrations of fisetin for 2 days after confluence. Total RNA ( $1 \mu \mathrm{g})$ was reverse transcribed with the Transcriptor First Strand cDNA Synthesis Kit (Roche Diagnostics K.K., Tokyo, Japan) with random primers in a $20-\mu \mathrm{L}$ reaction mixture according to the manufacturer's protocol. The quantitative PCR analysis was performed with a LightCycler 480 System II (Roche Diagnostics K.K.) and LightCycler 480 SYBR Green I Master (Roche Diagnostics K.K.). $\quad$ The PCR $\left(95^{\circ} \mathrm{C}\right.$ for $10 \mathrm{~s}, 55^{\circ} \mathrm{C}$ for $10 \mathrm{~s}$, and $72{ }^{\circ} \mathrm{C}$ for $10 \mathrm{~s}$, for 45 cycles $)$ was performed with specific primers (sense primer, 5'- GATGCACTGCCTATGAGCACTT- 3', and antisense primer, 5'- AGAGGTCCACAGAGCTGATTCC- 3') for mouse PPAR $\gamma$, (sense primer, 5'- CCGCAGACGACAGGAAGGT -3', and antisense primer, 5'AGGGCCCCGCCATCT -3') for mouse aP2, (sense primer, 5' CTCTGGGAAGCATCGAGAAG $-3^{\prime}$, and antisense primer, 5'GCATGGTGTGTCGAGAAAGA -3') for mouse perilipin, (sense primer, 5'TGGGCATCACCACGAAAAT -3', and antisense primer, 5'ATCAGCTGCACATCACTCAGAATT -3') for mouse 36B4, and (sense primer, 5'- 
TTCTACAATGAGCTGCGTGTG -3', and antisense primer, 5' GGGGTGTTGAAGGTCTCAAA -3') for mouse ACTB. 36B4 (for PPAR $\gamma$ and aP2) and ACTB (for perilipin) were used as internal standards for the normalization of each sample.

Statistical Analysis: The data are expressed as mean \pm standard deviation (SD). The statistical analysis was performed with Student's t-tests to compare the group means with and without fisetin administration. Two-tailed $P$-values less than 0.05 were considered statistically significant.

\section{RESULTS:}

Effects of fisetin on the adipogenetic differentiation of 3T3-L1 preadipocytes: During the differentiation of 3T3-L1 cells, the cells were treated with fisetin at 25 to $75 \mu \mathrm{M}$, and intracellular oil droplets were stained with Oil-red $\mathrm{O}$ and quantified. Fisetin at $50 \mu \mathrm{M}$ and 75 $\mu \mathrm{M}$ significantly suppressed lipid accumulation, and $75 \mu \mathrm{M}$ of fisetin inhibited it to $50 \%$ compared to dimethyl sulfoxide (DMSO)-treated control cells (Figs. 1A and 1B). Furthermore, to examine the suppressive effects of fisetin on differentiation, GPDH activity was measured in 3T3-L1 cells that were treated with fisetin (Fig. 1C). GPDH activity was completely inhibited by $75 \mu \mathrm{M}$ of fisetin.

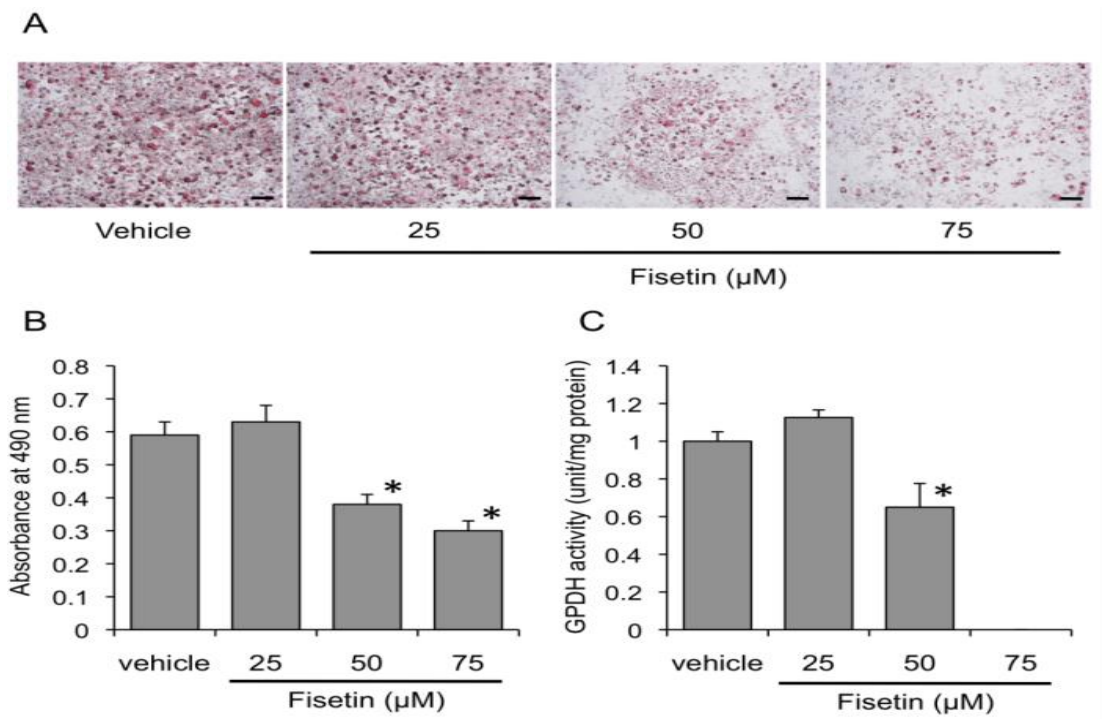

Figure 1. Fisetin suppressed intracellular lipid accumulation and GPDH activity in 3T3-L1 cells during differentiation. The cells were differentiated to adipocytes as described in the Materials and Methods section. During differentiation, $25 \mu \mathrm{M}, 50 \mu \mathrm{M}, 75 \mu \mathrm{M}$, or DMSO, which was used as a vehicle control, was added to the medium. (A) The cells were stained with Oil-red O. Bars $=100 \mu \mathrm{m}$. (B) Intracellular lipid accumulation was determined by Oil-red $\mathrm{O}$. The values are expressed as the mean \pm SD of 3 independent experiments. (C) Intracellular GPDH activity was measured. The values were calculated as a ratio of the vehicle and expressed as the mean \pm S.D. of 3 independent experiments. $* \mathrm{P}<0.05$ vs. vehicle. 
A

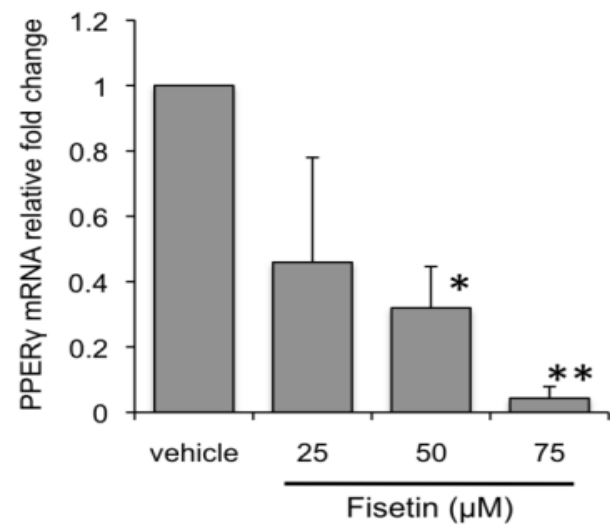

C

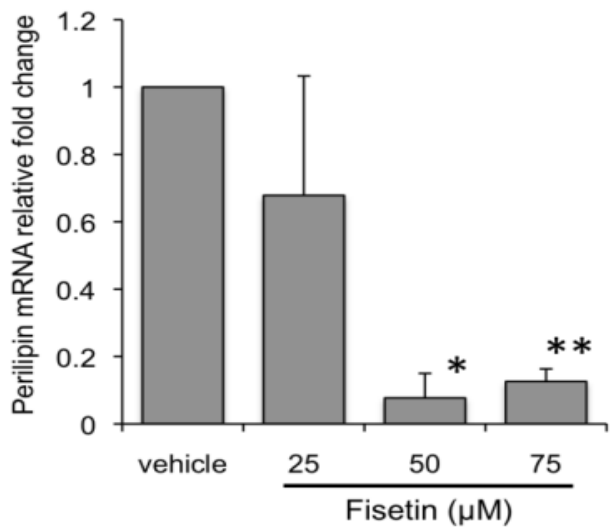

B

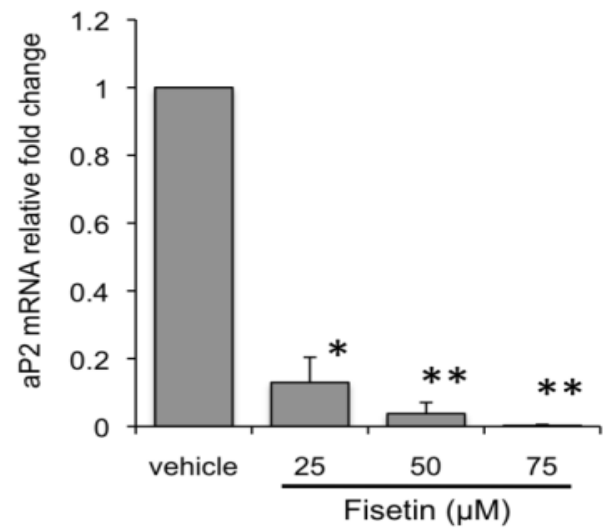

Figure 2. Fisetin suppressed the expression of $\operatorname{PPAR} \gamma, \mathrm{aP} 2$, and perilipin. The cells were differentiated to adipocytes as described in the Materials and Methods section. During differentiation, $25 \mu \mathrm{M}, 50 \mu \mathrm{M}, 75 \mu \mathrm{M}$, or DMSO (vehicle control) was added to the medium. The mRNA levels of PPAR $\gamma$ (A), aP2 (B), and perilipin (C) were determined by real-time PCR analysis. The values were calculated as a ratio of the vehicle and expressed as the mean \pm S.D. of 3 independent experiments. $* \mathrm{P}<0.05 v s$. vehicle, $* * \mathrm{P}<0.01 v s$. vehicle.

To investigate the inhibitory mechanisms of fisetin during adipocyte differentiation, we examined the expression of PPAR $\gamma$, which acts as a key transcription factor at an early stage of adipocyte differentiation, and the expression of aP2 and perilipin, both of which are expressed down-stream of PPAR $\gamma$. Real-time PCR revealed that fisetin suppressed the expression of PPAR $\gamma$, aP2, and perilipin mRNAs in 3T3-L1 cells in a dose dependent-manner (25 to $75 \mu \mathrm{M})$, as shown in Figs. 2A, 2B, and 2C, respectively.

Effects of fisetin on HFD-induced obesity: We examined the effects of fisetin on body weight in C57BL/6 female mice that were fed a control diet or HFD for 8 weeks. Fisetin ( $20 \mathrm{mg} / \mathrm{kg}$ body weight) was intraperitoneally injected into the mice of each group twice per week. Twenty $\mathrm{mg} / \mathrm{kg}$ body weight of fisetin did not affect the body weights of normal diet (ND) mice, but significantly increased the body weights of HFD-treated mice (Fig. 3A). Figure 3B shows typical CT images that were obtained with the 


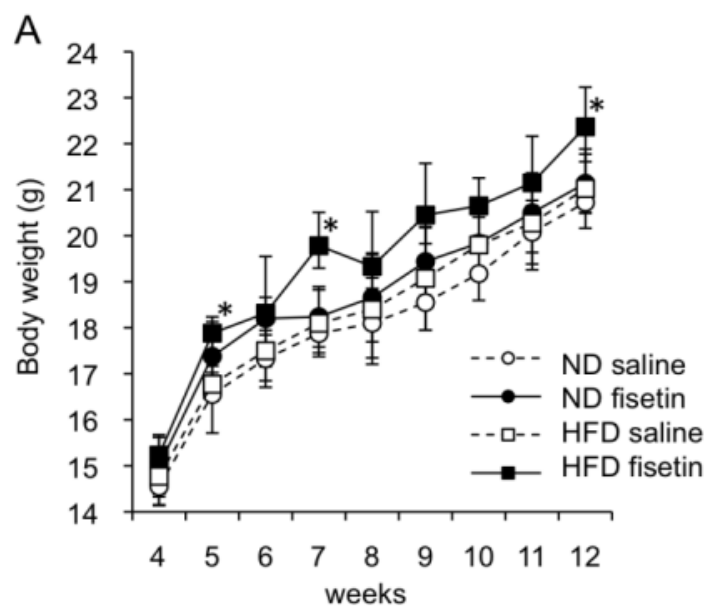

B
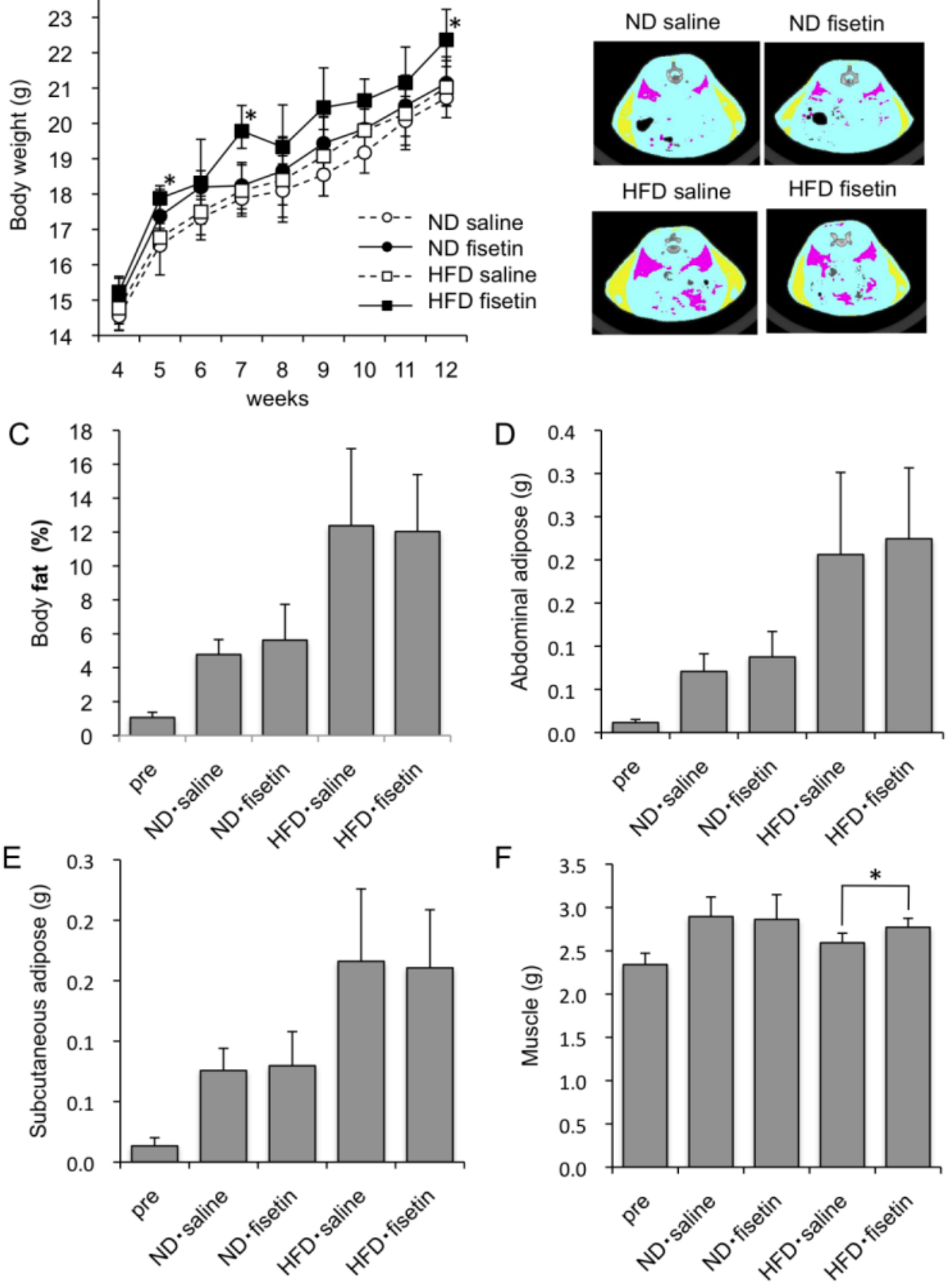

Figure 3. Fisetin did not show antiobesity activity in HFD-treated obese mice. Mice ( $\mathrm{n}=4$ or 5 per group) were treated with fisetin $(20 \mathrm{mg} / \mathrm{kg}$ body weight) by intraperitoneal injections twice per week for 8 weeks. (A) The body weights of the mice were measured. (B) Typical images of X-ray computed tomography of the mice abdomens for the detection of fat tissue. The detected tissue was assessed for body fat (C), abdominal adipose tissue (D), subcutaneous adipose tissue (E), and muscle (F). $\quad * \mathrm{P}<0.05$ vs. HFD saline.

LA Theta LCT-100 from the abdomen for the detection of fat tissue. Furthermore, the amounts of body fat (Fig. 3C), abdominal adipose tissue (Fig. 3D), subcutaneous adipose tissue (Fig. 3E), and muscle (Fig. 3F) were calculated for each group. Fisetin did not affect the fat tissues or muscle in both groups of mice fed a ND or HFD. 
A
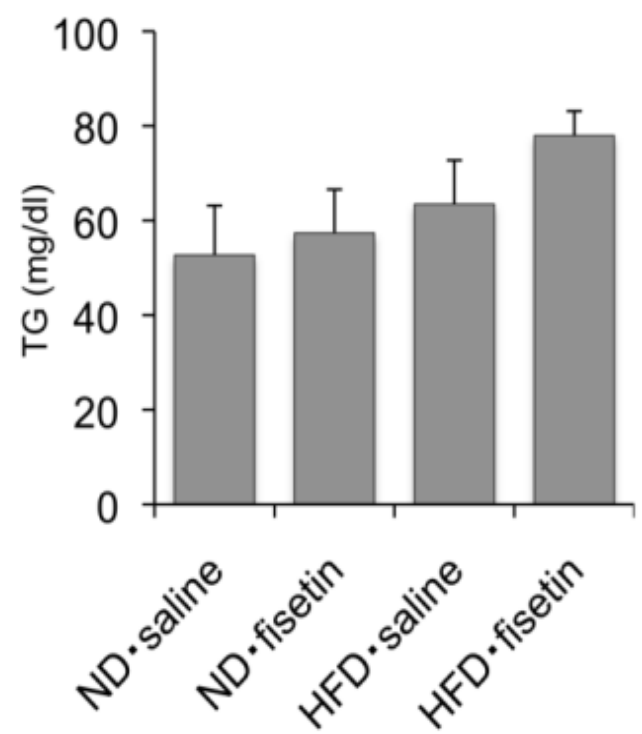

C

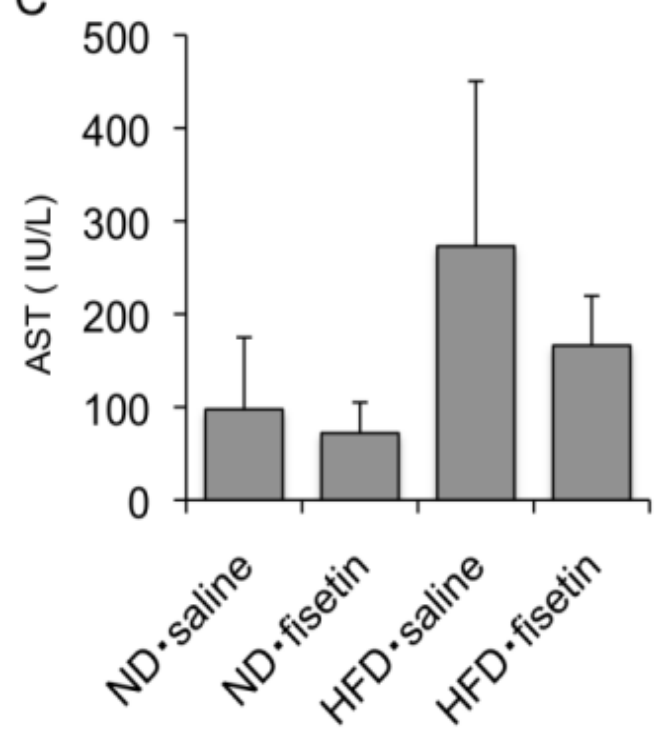

B

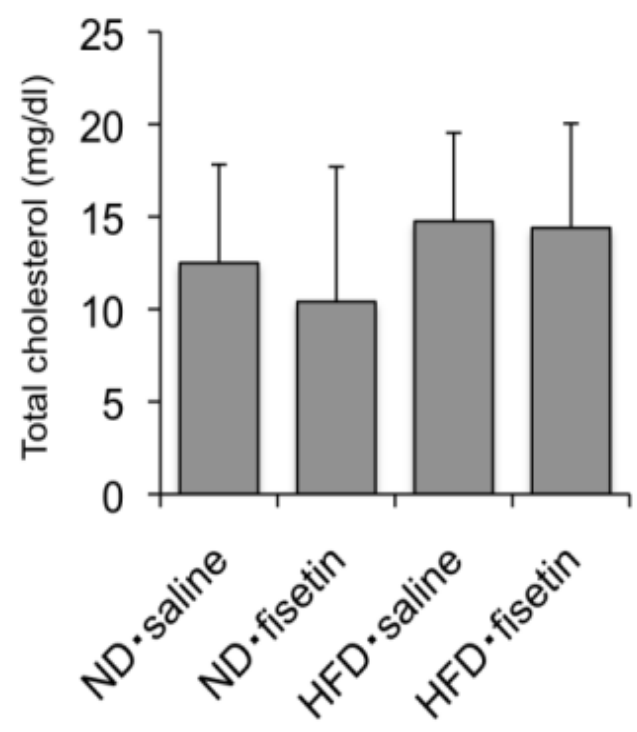

D

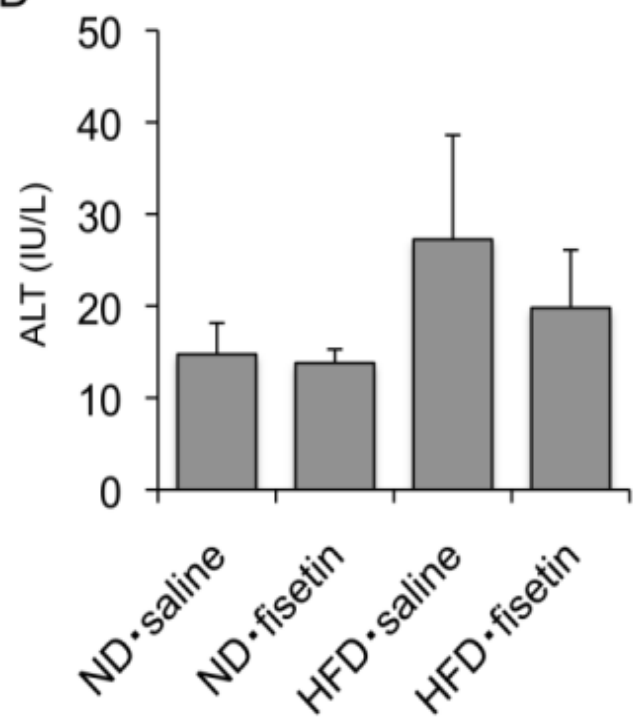

Figure 4. Fisetin did not improve obesity-related blood biochemical parameters. Blood samples from the mice were measured by BML, Inc. The levels of TG (A), total cholesterol (B), AST (C), and ALT (D) are expressed as the mean \pm SD.

Next, we monitored the levels of TG (Fig. 4A), total cholesterol (Fig. 4B), AST (Fig. 4C), and ALT (Fig. 4D) in the blood samples at the end of the 8 weeks of the HFD. Fisetin did not improve the obesity-related blood biochemical parameters in the HFD-treated obesity group.

As shown in Figure 5, the HFD-treated obese mice had fatty livers (Oil-red O stain), but fisetin did not suppress the fatty livers in the HFD-treated obese mice. Fisetin did not affect the kidneys of ND and HFD-treated obese mice. 

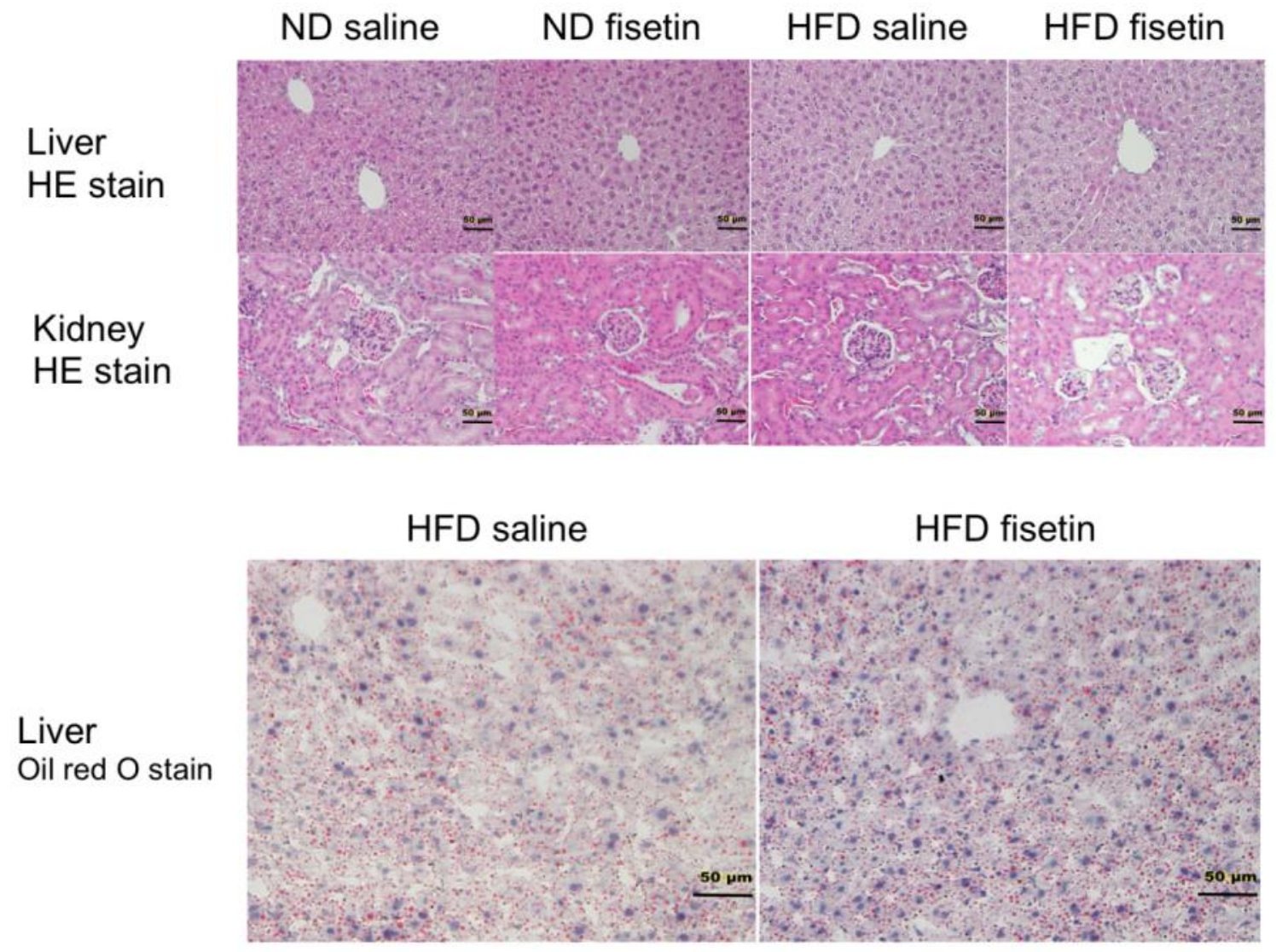

Figure 5. Fisetin did not suppress fatty liver in the HFD-treated obese mice. Liver tissues from the mice were analyzed histochemically with hematoxylin-eosin (HE) stain and Oil-red O stain. Kidnev tissues from the mice were also analvzed with HE stain. Bars $=50 \mathrm{um}$.

\section{DISCUSSION:}

In this study, we attempted to clarify the potential effects of the polyphenol fisetin on obesity, and found that fisetin attenuated adipocyte differentiation (intracellular lipid accumulation and GPDH activity) through the inhibition of the expression of PPAR $\gamma$ mRNA during the differentiation of 3T3-L1 preadipocytes. Furthermore, the injection of fisetin at a dose of 20 $\mathrm{mg} / \mathrm{kg}$ body weight into HFD-treated obese mice for 8 weeks increased the body weight, but it did not show anti-obesity effects on HFD-induced obesity. The effect of fisetin on weight gain in mice fed a HFD is interesting, although the underlying mechanism is unclear.

C57BL/6 male mice have been used to study HFD-induced obesity [19-22]. Recently, gender differences in weight gain have been studied in angiotensin receptor- [23], Nur77- [24], and lecithin cholesterol acyltransferase- (25) knockout mice with HFD-induced obesity. We used C57BL/6 female mice in this study because we attempted to investigate the effects of fisetin 
on alterations in abdominal adipose tissue in female mice in which it is difficult to accumulate abdominal adipose tissue compared to subcutaneous adipose tissue. CT scanning revealed that fisetin at $20 \mathrm{mg} / \mathrm{kg}$ body weight did not affect the fat tissues containing abdominal adipose tissue in both groups of mice fed ND and HFD.

Fisetin at 25 to $75 \mu \mathrm{M}$ inhibited the expression of PPAR $\gamma$ mRNA, which is a key transcription factor at an early stage of adipocyte differentiation, in 3T3-L1 cells. However, the injection of $20 \mathrm{mg} / \mathrm{kg}$ body weight fisetin into HFD-treated mice did not show anti-obesity effects. Recently, it was reported that daily fisetin supplementation displayed hypocholesterolemic effects in HFD Sprague-Dawley rats [26]. Future studies on high-dose fisetin in HFD-treated obese mice may aid the prevention of obesity. We also hypothesized from our results that fisetin may decompose in the body. Fisetin may be used as an effective remedy in the treatment of the symptoms of obesity if we are able to elucidate the mechanisms by which fisetin specifically acts upon PPAR $\gamma$ expression in adipocytes.

CONCLUSIONS: Our results suggested that the inhibition of PPAR $\gamma$ signaling by fisetin inhbited the adipocyte differentiation of 3T3-L1 preadipocytes. However, fisetin at $20 \mathrm{mg} / \mathrm{kg}$ body weight did not affect HFD-induced obesity. These findings provide insights into the development of tools for the prevention and treatment of obesity.

Author's contributions: All authors contributed to this study.

Acknowledgments: This work was supported by Grants-in-Aid for Scientific Research from the Ministry of Education, Science, Sports and Culture of Japan. We would like to thank Mr. Mareki Ohtsuji for his help in the histochemistry, Mrs. K. Nakata for assistance with the cell cultures and Mrs. K. Takada for mouse maintenance.

\section{REFERENCES:}

1. Cowherd RM, Lyle RE, McGehee RE Jr., Molecular regulation of adipocyte differentiation. Semin. Cell Dev. Biol., 1999; 10: 3-10.

2. Farmer SR, Transcriptional control of adipocyte formation. Cell Metab., 2006; 4: 263-273.

3. Furukawa S, Fujita T, Shimabukuro M, et al., Increased oxidative stress in obesity and its impact on metabolic syndrome. J. Clin. Invest., 2004; 114: 1752-1761. 
4. Saito T, Abe D, Sekiya K, Sakuranetin induces adipogenesis of 3T3-L1 cells through enhanced expression of PPAR 2 2. Biochem. Biophys. Res. Commun., 2008; 372: 835-839.

5. Gamo K, Miyachi H, Nakamura K, et al., Hesperetin glucuronides induce adipocyte differentiation via activation and expression of peroxisome proliferator-activated receptor- $\gamma$. Biosci. Biotechnol. Biochem., 2014; 78: 1052-1059.

6. Liu L, Shan S, Zhang K, et al., Naringenin and hesperetin, two flavonoids derived from Citrus aurantium up-regulate transcription of adiponectin. Phytother. Res., 2008; 22: 1400-1403.

7. Ahn J, Lee H, Kim S, et al., The anti-obesity effect of quercetin is mediated by the AMPK and MAPK signalling pathways. Biochem. Biophys. Res. Commun., 2008; 373: 545-549.

8. Kim H, Hiraishi A, Tsuchiya K, et al., (-) Epigallocatechin gallate suppresses the differentiation of 3T3-L1 preadipocytes through transcription factors FoxO1 and SREBP1c. Cytotechnology, 2010; 62: 245-255.

9. Prasad CNV, Mohan SS, Banerji A, et al., Kaempferitrin inhibits GLUT4 translocation and glucose uptake in 3T3-L1 adipocytes. Biochem. Biophys. Res. Commun., 2009; 380: $39-43$.

10. Snoussi C, Ducroc R, Hamdaoui MH, et al., Green tea decoction improves glucose tolerance and reduces weight gain of rats fed normal and high-fat diet. J. Nutr. Biochem., 2014; 25: 557-564.

11. Boque N, de la Iglesia R, de la Garza AL, et al., Prevention of diet-induced obesity by apple polyphenols in Wistar rats through regulation of adipocyte gene expression and DNA methylation patterns. Mol. Nutr. Food Res., 2013; 57: 1473-1478.

12. Wang L, Yamasaki M, Katsube T, et al., Antiobesity effect of polyphenolic compounds from molookheiya (Corchorus olitorius L.) leaves in LDL receptor-deficient mice. Eur. J. Nutr., 2011; 50: 127-133.

13. Khan N, Asim M, Afaq F, et al., A novel dietary flavonoid fisetin inhibits androgen receptor signalling and tumor growth in athymic nude mice. Cancer Res., 2008; 68: 8555-8563.

14. Lee JD, Huh JE, Jeon G, et al., Flavonol-rich RVHxR from Rhus verniciflua stokes and its major compound fisetin inhibits inflammation-related cytokines and angiogenic factor in rheumatoid arthritic fibroblast-like synovial cells and in vivo models. Int. Immunopharmacol., 2009; 9: 268-276. 
15. Leotoing L, Wauquier F, Guicheux J, et al., The polyphenol fisetin protects bone by repressing NF-KB and MKP-1-dependent signaling pathways in osteoclasts. PLoS One, 2013; 8, e68388.

16. Maher P, Akaishi T, Abe K, Flavonoid fisetin promotes ERK-dependent long-term potentiation and enhances memory. Proc. Natl. Acad. Sci. USA, 2006; 103: 16568-16573.

17. Hanneken A, Lin FF, Johnson J, et al., Flavonoids Protect Human Retinal Pigment Epithelial Cells from Oxidative-Stress-Induced Death. Invest. Ophthalmol. Vis. Sci., 2006; 47: 3164-3177.

18. Jung $\mathrm{CH}$, Kim $\mathrm{H}$, Ahn J, et al., Fisetin regulates obesity by targeting mTORC1 signaling. J. Nutr. Biochem., 2013; 24: 1547-1554.

19. Son Y, Nam J-S, Jang M-K, et al., Antiobesity activity of Vigna nakashimae extract in high-fat diet-induced obesity. Biosci. Biotechnol. Biochem., 2013; 77: 332-338.

20. Rustenbeck I, Lier-Glaubitz V, Willenborg M, et al., Effect of chronic coffee consumption on weight gain and glycaemia in a mouse model of obesity and type 2 diabetes. Nutr. Diabetes, 2014; 4: e123.

21. Balsevich G, Uribe A, Wagner KV, et al., Interplay between diet-induced obesity and chronic stress in mice: potential role of FKBP51. J. Endocrinol., 2014; 222: 15-26.

22. Evans CC, LePard KJ, Kwak JW, et al., Exercise prevents weight gain and alters the gut microbiota in a mouse model of high fat diet-induced obesity. PLos One, 2014; 9: e92193.

23. Samuel P, Khan MA, Nag S, et al., Angiotensin $\mathrm{AT}_{2}$ receptor contributes towards gender bias in weight gain. PLoS One, 2013; 8: e48425.

24. Perez-Sieira S, Martinez G, Porteiro B, et al., Female Nur77-deficient mice show increased susceptibility to diet-induced obesity. PLos One, 2013; 8: e53836.

25. Li L, Hossain MA, Sadat S, et al., Lecithin cholesterol acyltransferase null mice are protected from diet-induced obesity and insulin resistance in a gender-specific manner through multiple pathways. J. Bio. Chem., 2011; 286: 17809-17820.

26. Shin MJ, Cho Y, Moon J, et al., Hypocholesterolemic effect of daily fisetin supplementation in high fat fed Sprague-Dawley rats. Food Chem. Toxicol., 2013; 57: 84-90. 